\title{
Multi-UAV Cooperative Surveillance with Spatio-Temporal Specifications
}

\author{
Ali Ahmadzadeh, James Keller, George J. Pappas, Ali Jadbabaie and Vijay Kumar
}

\begin{abstract}
We present a path planning algorithm for timecritical cooperative surveillance using a set of unmanned aerial platforms. The unique constraints imposed by maneuver limits and body-fixed cameras make the problem quite challenging. An Integer Programming(IP)-based strategy for successfully operating within these constraints is developed. IP is applied over a receding planning horizon with terminal cost to reduce the computational effort of the planner and to incorporate feedback. The main contribution of the paper is the incorporation of highly constrained motion and sensor capabilities of the vehicles in the mathematical programming formalism. Simulation and experimental results are presented to demonstrate the efficacy of the proposed approach
\end{abstract}

\section{INTRODUCTION}

$\mathbf{T}$ HE use of Unmanned Aerial Vehicles (UAVs) equipped with cameras for surveillance is a natural but challenging application of robotic technology. Unlike ground robots, aerial platforms have many more operating constraints and their dynamic response typically dictates the nature of their role in such an application. [1]-[5]

There is a small class, electrically powered fixed-wing aerial vehicle that feature body-fixed cameras to provide a low cost platform with which to conduct surveillance operations. Their advantages are that they are portable, quiet, difficult to spot when aloft, inexpensive to acquire and operate. Fig. 1 illustrates one example of these UAVs recently developed at the GRASP Laboratory of the University of Pennsylvania. Each UAV consists of an airframe and engine, avionics package, onboard laptop and additional sensing payload [1].

We would like to develop a path planning capability to enable this class of vehicles to perform missions arguably outside of their design envelope. The benefit would be a lowcost, portable solution capable of being transported by ground personnel on foot. The system could, therefore, be considered to be mobile. The constraints imposed by selection of this type of air vehicle are summarized in Table I.

While the constraints summarized in Table I are not exact envelope limits, they should be considered as practical limits within which performance and trajectory control is reliable. These vehicles have inner loop controllers that provide the requisite stability and control for the navigation autopilots they use so that waypoint navigation using GPS is reliable spatially but temporal performance is affected by ambient winds.

Authors are with GRASP Laboratory, University of Pennsylvania, Philadelphia, PA 19104,USA

\{aliahmad, jfkeller, pappasg, jadbabai, kumar\}@grasp.cis. upenn.edu
TABLE I

THE CONSTRAINTS OF SMALL, ELECTRICALLY POWERED FIXED-WING AERIAL VEHICLE THAT FEATURES BODY-FIXED CAMERAS

\begin{tabular}{|c|c|}
\hline Speed (airspeed) & Fixed in $10-20 \mathrm{~m} / \mathrm{s}$ range \\
\hline $\begin{array}{l}\text { Minimum Turning } \\
\text { Radius }\end{array}$ & $\begin{array}{l}\text { On order of } 40 \text { to } 150 \mathrm{~m} \text { (normal load factor } \\
\leq 1.05 \mathrm{~g} \text { in level flight) }\end{array}$ \\
\hline Flight Characteristics & Constant airspeed sideslip Control \\
\hline Degrees of Freedom & 2 to 3 \\
\hline Dynamic Response & 1 to $2 \mathrm{sec}$ to transition to turning flight \\
\hline Camera Geometry & $\begin{array}{l}\text { Field of View: } 20-45 \text { deg, fixed line of } \\
\text { sight(forward, to left, or right) }\end{array}$ \\
\hline
\end{tabular}

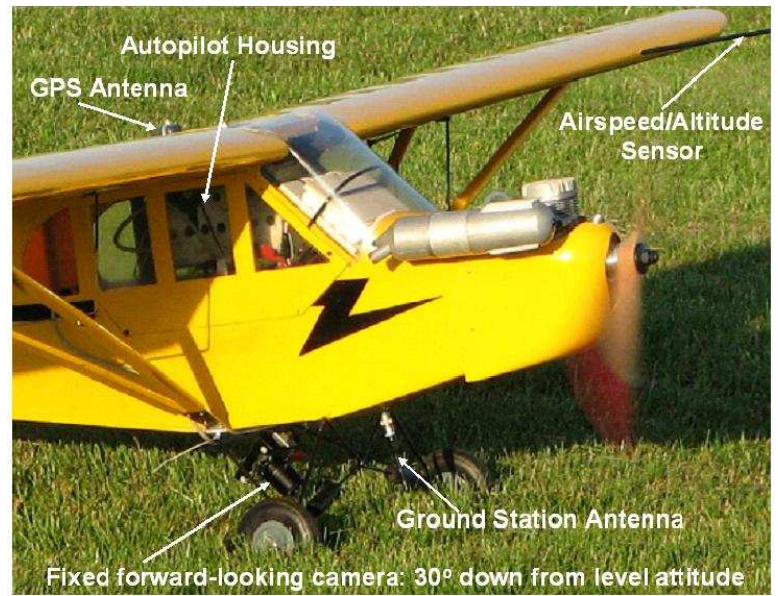

Fig. 1. PennUAVs: External Payloads (POD)

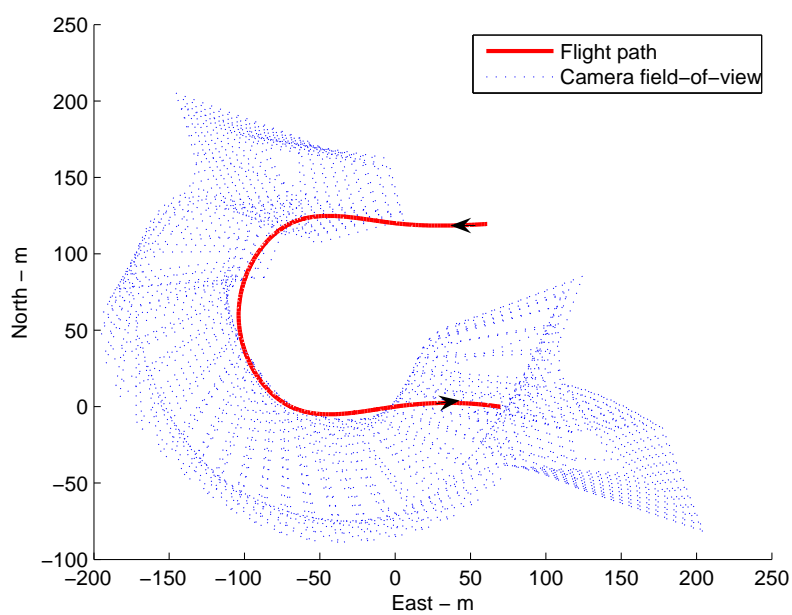

Fig. 2. Front Camera field-of-view on ground plane 


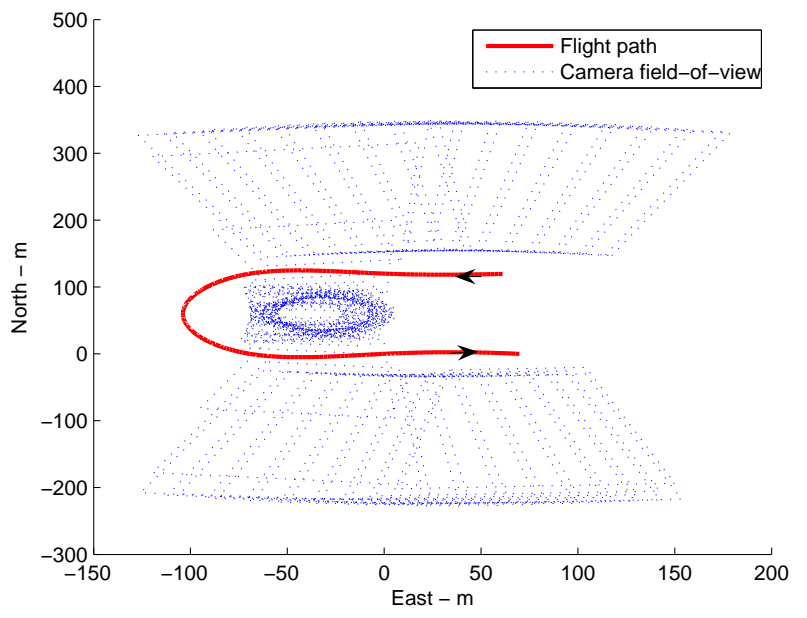

Fig. 3. Left Camera field-of-view on ground plane

The nonlinear behavior of the field of view of the camera mounted on these UAVs makes the coverage problem even more challenging. The coupling between flight path and footprint of the forward-looking camera is illustrated in the Fig. 2 , which also depicts how the shape of the camera frame on the ground-plane is distorted during turning flight. Similarly, Fig. 3 illustrates the same concepts for a left-looking camera.

This paper studies the cooperative motion-planning problem for a group of heterogeneous UAVs which have to monitor, surveil and generate mosaic maps of their operations area periodically. Each UAV is modeled as a nonholonomic point mass moving on a two-dimensional (2-D) plane at a constant speed with a minimum turning radius. This model is also known as Dubins car in the literature [6]. There exists extensive research on multi-UAV task scheduling and planning which doesn't address the coverage problem. For a review the interested reader can refer to [10], [11]. One approach to the coverage problem is based on cellular decomposition. When using a cellular decomposition either each cell is covered when the vehicle crosses it or the vehicle has to perform some specific motion pattern (usually a boustrophedon motion) in order to cover the cell [12]. This method mainly focuses on the single robot coverage and nonholonomic constraints are not considered.

Most of prior work in the area of coverage concentrated on sensor network problem [13]-[16]. The cooperation of multiple sensors is often achieved by choosing different sensors (or sensor modes) for different tasks (targets) at different times and motion planning is not involved in these problems. Cortes et al. [17] studied the multi-sensor localization problem in a polygonal environment and developed a gradient-descent algorithm for optimal coverage and sensing policies. Each sensor agent is expected to converge to its optimal location and stay there. Enrigh et al. [18] considered the problem of visiting stochastically-generated targets in a planar bounded region with the objective of minimization of the expected waiting time between the appearance of a target, and the time it is visited.

\section{PRoblem Definition}

The general problem of interest can be described as follows: Given a closed and bounded region $\Omega \subset \Re^{2}$ and $N$ heterogenous UAVs $\left\{u_{1}, u_{2}, \ldots, u_{N}\right\}$, each equipped with a fixed mount camera and a refresh time $\tau$, find feasible trajectories $\gamma_{i}(t), i=1, \ldots, N$ for UAVs $u_{i}, i=1, \ldots, N$ in order to get maximum coverage of the region $\Omega$ in each time interval $[n \tau,(n+1) \tau], n=0,1, \ldots$

The point $p \in \Omega$ is covered in given time interval $[k \tau,(k+$ 1) $\tau]$ if there exist time $t \in[k \tau,(k+1) \tau]$ such that $p$ is in the field of view of at least one UAV. The coverage of $\Omega$ in each length $\tau$ time interval crucially depends on the initial states of UAVs at the beginning of the time interval, the field of view of UAVs and the number of the vehicles $N$. As a result, full coverage of the set $\Omega$ may not be feasible in each length $\tau$ time intervals.

We assume that all UAVs fly with fixed and distinct altitudes. The UAVs are modeled as nonholonomic vehicles constrained to move at constant speed $v_{i} i=1 \ldots N$ along a path with bounded curvature.

Throughout the paper we use the following notations. Consider an n-tuple $\alpha=\left(\alpha_{1}, \ldots, \alpha_{n}\right)$ of nonnegative integers, then we define the sum as $[\alpha]=\sum \alpha_{i}$ and $\frac{\partial^{\alpha}}{\partial x^{\alpha}}=\frac{\partial^{[\alpha]}}{\partial x_{1}^{\alpha_{1}} \ldots \partial x_{n}^{\alpha_{n}}}$. Let $f: \mathfrak{R}^{n} \rightarrow \mathfrak{R}$. We say that $f$ is class $C^{k}$ for nonnegative integer $k$, if the partial derivatives $\frac{\partial^{\alpha}}{\partial x^{\alpha}}$ exist and are continuous for $[\alpha] \leq k$. If $f: \mathfrak{R}^{n} \rightarrow \mathfrak{R}^{m}$, then $f$ is class $C^{k}$ if each of the component functions $f_{i}$ is class $C^{k}$. Also $\|$.$\| denote Euclidian$ norm.

For the set $V$, measure $\mu(V)$ is defined as:

$$
\mu(V)=\int_{V} \chi_{v}(x) d x
$$

where $\chi_{v}(x)$ is indicator function defined as:

$$
\chi_{v}(x)= \begin{cases}1 & x \in V \\ 0 & x \notin V\end{cases}
$$

For the set $V \subset \mathfrak{R}^{2}$, let $M(V)$ be the mass center of the inertia as:

$$
M(V)=\int_{V} x d x
$$

A planar path or trajectory is a function $\gamma:\left[t_{0}, t_{1}\right] \rightarrow \mathfrak{R}^{2}$ as $\gamma_{i}(t)=\left(x_{i}(t), y_{i}(t)\right)$. Signed curvature of the path $\gamma(t)$ is $\kappa(t)$ and is defined as:

$$
\kappa(t)=\left(x(t)^{\prime} y(t)^{\prime \prime}-x(t)^{\prime \prime} y(t)^{\prime}\right) /\left\|\gamma^{\prime}(t)\right\|^{3}
$$

A planar trajectory $\gamma_{i}(t)$ is called flyable by vehicle $u_{i}$ if it is class $C^{2}$ and the absolute value of the signed curvature of the trajectory is always less than $1 / \rho_{i}$ where $\rho_{i}$ is the minimum radius of a flyable circle by UAV $u_{i}$. Since the trajectory $\gamma_{i}(t)$ of UAV $u_{i}$ is a planar curve with constant speed $v_{i}$, signed curvature function $\kappa_{i}(t)$ and initial conditions $\gamma_{i}\left(t_{0}\right)$ and $\theta_{i}\left(t_{0}\right)$ are sufficient to fully specify trajectory $\gamma_{i}(t)$ as described in the following theorem adapted from [19].

Theorem 1: Let $\kappa:\left[t_{0}, t_{0}+\tau\right] \rightarrow[-1 / \rho, 1 / \rho]$ be a piecewise continuous curvature function for the planar trajectory $\gamma(t)$ with given initial conditions $\gamma\left(t_{0}\right)=\left(x\left(t_{0}\right), y\left(t_{0}\right)\right), \theta\left(t_{0}\right)$ and 
constant speed $\left\|\gamma_{i}^{\prime}(t)\right\|=v_{i}$. Then the parameterized trajectory $\gamma:\left[t_{0}, t_{0}+\tau\right] \rightarrow \mathfrak{R}^{2}$ can be written as:

$$
\gamma(t)=(x(t), y(t))
$$

where

$$
\begin{aligned}
& x(t)=v \int_{t_{0}}^{t} \cos (\theta(\zeta)) d \zeta+x\left(t_{0}\right) \\
& y(t)=v \int_{t_{0}}^{t} \sin (\theta(\zeta)) d \zeta+y\left(t_{0}\right)
\end{aligned}
$$

and

$$
\theta(\zeta)=\int_{t_{0}}^{\zeta} \kappa(\xi) d \xi+\theta\left(t_{0}\right)
$$

Also $\gamma(t)$ is a class $C^{2}$ and flyable trajectory for a UAV with constant speed $v$ and initial conditions $\gamma\left(t_{0}\right)$ and $\theta\left(t_{0}\right)$ with minimum turning radius $\rho$.

This theorem enables us to change the search space from flyable planar trajectories $\gamma_{i}(t) i=1, . ., N$ to bounded scalar functions $\kappa_{i}(t) i=1, . ., N$.

Therefore we can restate our objective as generating curvature functions $\left\{\kappa_{i}(t)|| \kappa_{i}(t) \mid \leq 1 / \rho_{i}, i=1,2, \cdots, N\right\}$ for all UAVs in order to get maximum coverage for all of the length $\tau$ time intervals $[n \tau,(n+1) \tau], n=0,1, \ldots$

\section{Modeling of the CAmera Field of VieW}

Each UAV is equipped with either a left or a front looking camera, mounted on the left wing or front of the vehicle respectively. Cameras are both looking at the ground with a fixed angle. Since UAVs have to bank to turn, the lookdown angle of the cameras change when UAVs turn. As it has been illustrated in Fig. 4 the field of view of the front camera expands when vehicle turns (left or right) and the field of view of left camera shrinks when vehicle turns left. When the vehicle turns right too much, the camera faces the horizon and doesn't see anything.

The field of view of each camera (left or front) at any time is a function of position, heading (yaw) and bank angle (roll) of the vehicle. Moreover for a given vehicle $u_{i}$ and a fixed bank angle, the field of view for different positions and orientations differ by a rigid motion.
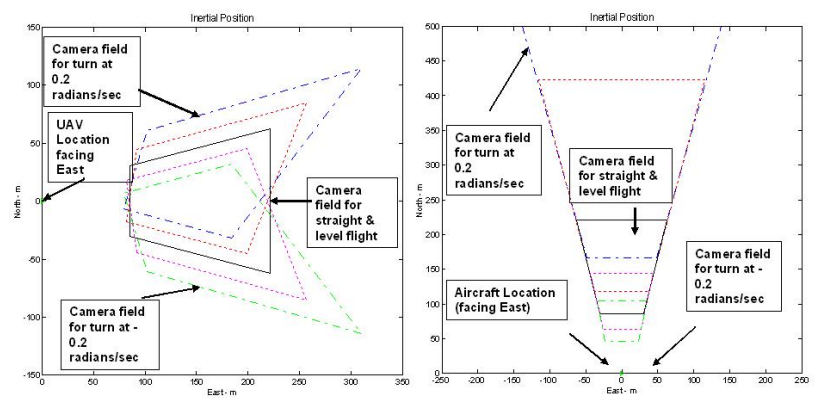

Fig. 4. Front and Left Camera field-of-view on ground plane for turn rates of $+0.2 \mathrm{radians} / \mathrm{sec}$ in increments of $0.1 \mathrm{radians} / \mathrm{sec}$

Assume that $\theta_{i}(t)$ is the heading of the vehicle $u_{i}$ with respect to the $y=0$ axis. Also let the bank angle of the vehicle at time $t$ be $\phi_{i}(t)$. As we mentioned earlier for each vehicle $u_{i}$ at given time $t$, the position of vehicle $\gamma_{i}(t)$, heading of the vehicle $\theta_{i}(t)$ and the bank angle $\phi_{i}(t)$ are sufficient to uniquely specify the field of view.

In the following, we introduce an alternative approach to uniquely specify field of view of each UAV.

With assumption of the coordinated level turn, bank angle $\phi_{i}(t)$ is an explicit function of the curvature of the path $\gamma_{i}(t)$ and the speed of vehicle at time $t$ and is given as follows:

$$
\phi_{i}(t)=\arctan \left(v_{i}^{2} \kappa_{i}(t) / g\right)
$$

Where $g$ is the gravity acceleration.

The heading of each vehicle $\theta_{i}(t)$ can be written as:

$$
\theta_{i}(t)=\arctan \left(y_{i}(t) / x_{i}(t)\right)
$$

Given any time instant $t_{0}$, the values of $\gamma_{i}\left(t_{0}\right), \gamma_{i}^{\prime}\left(t_{0}\right)$ and $\kappa_{i}\left(t_{0}\right)$ uniquely specify the field of view at time $t_{0}$. Based on this we define the state of configuration of the $\mathrm{N}$-vehicle system as follows.

Definition 1 (state of the system): The state of $\mathrm{N}$-vehicle system $\left\{u_{1}, u_{2}, \ldots, u_{N}\right\}$ with given trajectories $\left\{\gamma_{1}, \ldots, \gamma_{N}\right\}$ at any time $t$ is defined by a $N \times 5$ matrix as:

$$
s(t)=\left(\begin{array}{ccc}
\gamma_{1}(t) & \gamma_{1}^{\prime}(t) & \kappa_{1}(t) \\
\vdots & \vdots & \vdots \\
\gamma_{N}(t) & \gamma_{N}^{\prime}(t) & \kappa_{N}(t)
\end{array}\right)=\left(\begin{array}{c}
s_{1}(t) \\
\vdots \\
s_{N}(t)
\end{array}\right)
$$

As illustrated in Figure 4, the field of view for any bank angle is a bounded and convex polygonal set. Let field of view of vehicle $u_{i}$ with trajectory $\gamma_{i}(t)$ at time $t_{0}$ be given by $\psi_{i}\left(s_{i}\left(t_{0}\right)\right) \subset \mathfrak{R}^{2}$. Since $\psi_{i}\left(s_{i}\left(t_{0}\right)\right)$ is a convex polygon, there exist matrices $A\left(s_{i}\left(t_{0}\right)\right)$ and $b\left(s_{i}\left(t_{0}\right)\right)$ such that:

$$
\psi_{i}\left(s_{i}\left(t_{0}\right)\right)=\left\{p \in \mathfrak{R}^{2} \mid A\left(s_{i}\left(t_{0}\right)\right) p \leq b\left(s_{i}\left(t_{0}\right)\right)\right\}
$$

We assume that matrices $A\left(s_{i}\left(t_{0}\right)\right)$ and $b\left(s_{i}\left(t_{0}\right)\right)$ are given for the configurations, where vehicle $u_{i}$ is located at the origin with orientation $\theta_{i}\left(t_{0}\right)=0$ (see Figure 4) and for all admissible curvatures. For the configuration, where $u_{i}$ located at $\gamma(t)$ with orientation $\theta_{i}(t)$ we have:

$$
\begin{gathered}
A\left(s_{i}(t)\right)=A\left(s_{i}\left(t_{0}\right)\right) Q\left(-\theta_{i}(t)\right) \\
b\left(s_{i}(t)\right)=b\left(s_{i}\left(t_{0}\right)\right)-A\left(s_{i}(t)\right) \gamma(t)
\end{gathered}
$$

Where the curvature of $s_{i}\left(t_{0}\right)$ is equal to curvature of $s_{i}(t)$ and $Q$ is a rotation matrix as:

$$
Q(\theta)=\left(\begin{array}{cc}
\cos (\theta) & -\sin (\theta) \\
\sin (\theta) & \cos (\theta)
\end{array}\right)
$$

Definition 2 (Coverage map $\Psi$ ): At any time $t$ coverage map is mapping from the configuration space to the set $\Omega$ as follows:

$$
\Psi(s(t))=\bigcup_{i=1}^{N} \psi_{i}\left(s_{i}(t)\right) \bigcap \Omega
$$

Note that $\Psi(s(t))$ is actually a snapshot of the coverage of $\Omega$ at time $t$.

Now that we developed sufficient notations, In the following, we try to explore different plausible mathematical programming formulation of the coverage problem. The objective of the coverage problem is to find admissible curvature functions $\kappa_{i}(t) i=1 \ldots N$ to get maximum coverage of the region $\Omega$ 
in each length $\tau$ time interval. Let $\bar{\Omega}_{n}$ denote the uncovered region in the time interval $[n \tau,(n+1) \tau]$ as:

$$
\bar{\Omega}_{n}=\Omega-\bigcup_{t} \Psi(s(t)) \quad t \in[n \tau,(n+1) \tau]
$$

Note that in (18) the union is over the interval $[n \tau,(n+1) \tau]$ and the union set is not necessary a convex set.

Assumption 1: For any set of uncovered region $\bar{\Omega}_{n}$, we assume that there exist an $\varepsilon>0$, such that either $\mu\left(\bar{\Omega}_{n}\right)>\varepsilon$ or $\mu\left(\bar{\Omega}_{n}\right)=0$. Similarly we make the same assumption for the intersection of the two sets $\bar{\Omega}_{n} \cap \bar{\Omega}_{m}$.

Given the time interval $[n \tau,(n+1) \tau]$ and initial state $s(n \tau)$ we can state the coverage problem in the time interval as follows:

$$
\arg \min _{\kappa_{1}(t) \ldots \kappa_{N}(t)}\left(\mu\left(\bar{\Omega}_{n}\right)\right)
$$

The time horizon in the above optimization problem is $\tau$, but the objective is to maximize the coverage of the set $\Omega$ in all of the length $\tau$ time periods. Since the optimization is just for one period, at the end of the time period the final configuration of the $\mathrm{N}$-vehicle may be such that, it makes it impossible to recover the area in the next $\tau$ period. Therefore we modify the cost to be the upper bound asymptotic of the average area of uncovered region and the corresponding optimization problem is:

$$
\arg \inf _{\kappa_{1}(t) \ldots \kappa_{N}(t)}\left\{\limsup \left(\frac{1}{n} \sum_{i=0}^{n} \mu\left(\bar{\Omega}_{i}\right)\right)\right\}
$$

The above optimization actually tries to minimize the average value of area of uncovered regions in the time periods $[n \tau,(n+$ 1) $\tau] n=0,1, \ldots$. The drawback of this way of modeling the problem is that the cost function is forgetful, in the sense that if some part of the region is not covered in one time period, in the next time period this region won't have any priority over other covered regions. Therefore it is possible that some areas won't be covered at all, In the following, we propose a different cost function for solving this problem.

For any $p \geq q \geq 0$, let $\bar{\Omega}_{p, q}$ be defined as:

$$
\bar{\Omega}_{p, q}=\bigcap_{i=q}^{p} \bar{\Omega}_{i}
$$

Definition 3: For any $p \geq 0$ the function $\ell(p)$ is the minimum $q \geq 0$ such that $\bar{\Omega}_{p, q} \neq \emptyset$, therefore $\bar{\Omega}_{p, \ell(p)} \neq \emptyset$ and $\bar{\Omega}_{p, \ell(p)-1}=\emptyset$.

Now we define the coverage cost and the corresponding coverage optimization problem as the following:

$$
\begin{array}{r}
\mathrm{C}\left(\kappa_{1}(t), \ldots, \kappa_{N}(t)\right)=\limsup _{n \rightarrow \infty}\left\{\left[\frac{1}{n} \sum_{p=0}^{n} \mu\left(\bar{\Omega}_{p}\right)\right]\right. \\
\left.+\max _{1 \leq k \leq n}\left[(k-\ell(k)) \mu\left(\bar{\Omega}_{k, \ell(k)}\right)\right]\right\} \\
\arg \inf _{\kappa_{1}(t) \ldots \kappa_{N}(t)} \mathrm{C}\left(\kappa_{1}(t), \ldots, \kappa_{N}(t)\right)
\end{array}
$$

Theorem 2: The cost function in (21) is finite if and only if there exist $M>0$ such that

$$
\sup _{p \rightarrow \infty}(p-\ell(p)) \leq M
$$

Corollary 1: Given a set of trajectories for UAVs, the cost function (21) is finite if and only if, every point in set $\Omega$ will be revisited by at least one UAV in finite time.

In the following, we try to approximate the infinite horizon optimization problem in (22) with finite horizon optimization with terminal cost.

Control Lyapunov Functions have been used successfully as terminal penalties similar to method used in receding horizon control strategy [21] for Model Predictive Control (MPC), but MPC is incompatible with our problem setting since the cost function is not in the standard form (is union rather than integral). We consider a slightly modified optimization problem where the planning horizon at each time period is $[n \tau,(n+1) \tau]$ and we show that the solution of this modified optimization problem is a feasible (finite) solution for (21).

Definition 4: Given $s(t)$ the state of the system defined in the Def. 1 and a point $O \in \Re^{2}$, we define Dubins metric $L(s(t), O)$, the resulting cost of the shortest Dubins path from $s(t)$ to $O$. Note that Dubins metric is not, however, a true metric because it violates the symmetry axiom [22].

Please note that we are actually interested in the position of the $M\left(\psi_{i}\left(s_{i}(t)\right)\right.$ but $M\left(\psi_{i}\left(s_{i}(t)\right)\right.$ is simply translation and rotation of UAV's position. Also let's define $U$ as the maximum area disk that lies inside $\bar{\Omega}_{p, \ell(p)}$ and we can assume without loss of generality that $M(U)=O$.

Now we can state the finite horizon time coverage as the following optimization problem for the time period $[p \tau,(p+$ 1) $\tau]$

$$
\begin{array}{r}
\arg \min _{\kappa_{1}(t) \ldots \kappa_{N}(t)}\left\{\mu\left(\bar{\Omega}_{p}\right)+(p-\ell(p)) \mu\left(\bar{\Omega}_{p, \ell(p)}\right)\right. \\
\left.+\sum_{i=1}^{N} w_{i}(p+1-\ell(p)) L\left(s((p+1) \tau), M\left(\bar{\Omega}_{p, \ell(p)}\right)\right)\right\}
\end{array}
$$

Where $w_{i}>0 i=1 \ldots N$ are the weight coefficients.

Theorem 3: The control policy generated by solving optimization (24) generates a feasible (bounded) solution for (21).

Even though we reduced the problem of infinite horizon optimization to finite horizon optimization problems, due to the nonlinear and nonconvex nature of the problem, Solving (24) is still a very challenging problem. In the next section we propose some discretization techniques for solving optimization (24).

\section{IP FORMULATION BY DISCRETIZATION OF THE SPACE,TIME AND CURVATURE}

In the following, we try to solve (24) using discretization in the set $\Omega \in \mathfrak{R}^{2}$, curvature functions $\kappa_{i}$ and finally time.

\section{A. Discretization of $\Omega$ and time}

We partition the search space $\Omega$ into regions, using circles with radius $r$. The tessellation of the field to be observed is based on the geometry of the least capable camera and also the required resolution.

Let $B_{r}(p)$ represent the ball of radius $r$ and center $p$ as

$$
B_{r}(p)=\left\{q \in \mathfrak{R}^{2} \mid\|p-q\|_{2} \leq r\right\}
$$


The collection $\mathscr{C}=\left\{B_{r}\left(p_{1}\right), \ldots, B_{r}\left(p_{n}\right)\right\}$ is said to cover $\Omega$, or to be a covering of $\Omega$ if $\Omega \subseteq \bigcup_{i=1}^{n} B_{r}\left(p_{i}\right)$.

Given a covering $\mathscr{C}$, the discretized $\Omega$ or $\Omega^{d}$ is defined as the following set:

$$
\Omega^{d}=\left\{B_{r}(p) \in \mathscr{C} \mid B_{r}(p) \bigcap \Omega \neq \emptyset\right\}
$$

There exist methods for finding the minimum number of disks of radius $r$ to cover region $\Omega$ in the plane [19], however the number of the covering doesn't much effect the complexity of the coverage algorithm.

Similarly we define the discretized field of view $\psi_{i}^{d}\left(s_{i}(t)\right)$ as follows:

$$
\psi_{i}^{d}\left(s_{i}(t)\right)=\left\{B_{r}(p) \in \Omega^{d} \mid B_{r}(p) \subseteq \psi_{i}\left(s_{i}(t)\right)\right\}
$$

The following theorem offers an effective and computationally cheap way for computing $\psi_{i}^{d}\left(s_{i}(t)\right)$.

Theorem 4: Consider $\psi_{i}\left(s_{i}(t)\right)$ as

$$
\psi_{i}\left(s_{i}(t)\right)=\left\{q \in \mathfrak{R}^{2} \mid A_{i}\left(s_{i}(t)\right) q \leq b_{i}\left(s_{i}(t)\right)\right\}
$$

sufficient condition for $B_{r}(p) \in \psi_{i}^{d}\left(s_{i}(t)\right)$ is that

$$
A_{i}\left(s_{i}(t)\right) p \leq b_{i}\left(s_{i}(t)\right)-r \varepsilon \mathbf{1}
$$

where $\mathbf{1}$ is a vector of ones and:

$$
\varepsilon=\left\|A_{i}\left(s_{i}(t)\right)\right\|_{\infty}=\max _{j}\left(\left|a_{j 1}\right|+\left|a_{j 2}\right|\right)
$$

We also define discretized coverage map $\Psi^{d}(t)$ as :

$$
\Psi^{d}(s(t))=\bigcup_{i=1}^{N} \psi_{i}^{d}\left(s_{i}(t)\right)
$$

The discretization of the time interval $[n \tau,(n+1) \tau]$ is the set $T(n)=\left\{t_{i} \mid t_{i}=n \tau+\frac{i \tau}{z}, i=0, \ldots, z\right\}$.

The sets $\bar{\Omega}_{n}^{d}$ and $\bar{\Omega}_{p, q}^{d^{2}}$ are defined as:

$$
\bar{\Omega}_{n}^{d}=\Omega^{d}-\bigcup_{t_{k} \in T(n)} \Psi^{d}\left(s\left(t_{k}\right)\right)
$$

and

$$
\bar{\Omega}_{p, q}^{d}=\bigcap_{i=q}^{p} \bar{\Omega}_{i}^{d}
$$

\section{B. Discretization of curvature $\kappa_{i}$}

Since the range of the function $\kappa_{i}:\left[t_{0}, t_{1}\right] \rightarrow\left[-1 / \rho_{i}, 1 / \rho_{i}\right]$ is bounded, we can fairly approximate function $\kappa_{i}(t)$ with piecewise constant function $\kappa_{i}^{d}(t)$ as:

$$
\kappa_{i}^{d}:\left[t_{0}, t_{1}\right] \rightarrow K(\rho)
$$

Where

$$
K(\rho)=\left\{\kappa_{i} \mid \kappa_{i}=\frac{i}{m \rho}, i=-m,-m+1, \ldots, m\right\}
$$

Also we assume that curvature is constant for a time interval with length $\delta_{1}$. In order to make $\kappa_{i}^{d}(t)$ a continuous function, we assume that the change of curvature between two different values $k_{j}$ to $k_{l}$ is linear in time and takes $\delta_{2}$ sec. The transition between $k_{j}$ to $k_{l}$ is possible iff $|l-j| \leq 1$. Note that $\delta_{1}$ and $\delta_{2}$ are actually selected based on dynamic response of the UAVs.

\section{Integer Programming Formulation}

This section presents a Integer Programming (IP) formulation for the time critical cooperative coverage problem by discretization of (24) using discretized curvature and discretized field of view.

To proceed, assume that time is discretized. At any time (stage) $\mathrm{t}$, the decision (control) $\mathscr{U}(t)=\left(\kappa_{1}^{d}\left(t_{i}\right), \ldots, \kappa_{N}^{d}\left(t_{i}\right)\right)$ is defined to be a vector of constant curvatures for N-vehicle system. Policies are $\pi=\left\{\mu_{0}, \mu_{1}, \ldots, \mu_{z}\right\}$, where $\mu_{k}$ maps states $s\left(t_{k}\right)$ into controls $\mathscr{U}\left(t_{j}\right)=\mu_{j}\left(s\left(t_{j}\right)\right)$. The discretized coverage problem for the time interval $[p \tau,(p+1) \tau]$ is defined as the following IP:

$$
\begin{aligned}
& \arg \min _{\kappa_{1}^{d}\left(t_{i}\right) \ldots \kappa_{N}^{d}\left(t_{i}\right)}\left\{\operatorname{card}\left(\bar{\Omega}_{p}^{d}\right)+(p-\ell(p)) \operatorname{card}\left(\bar{\Omega}_{p, \ell(p)}^{d}\right)\right. \\
& \quad+\sum_{i=1}^{N} w_{i}(p+1-\ell(p)) L\left(s((p+1) \tau), M\left(\bar{\Omega}_{p, \ell(p)}^{d}\right)\right\}
\end{aligned}
$$

\section{Simulation Results}

The cooperative coverage algorithm that is presented in this paper has been successfully implemented and flight tested for surveillance. Also In the following, we show some simulation and implementation results based on the presented algorithm.

In the simulations, we consider a fleet of four vehicles operating at four distinct height-above-ground tiers. The objective is to cover a $400 \times 300 \mathrm{~m}^{2}$ area with the refresh time of $\tau=15 \mathrm{sec}$. We consider fields of view of 30 degrees for the cameras. As webcam type cameras can be used, a 4 x 3 aspect ratio is assumed, with the wide dimension orthogonal to the pitch attitude of the camera. The flight altitudes are 50, 75, 100 and $110 \mathrm{~m}$. We considered 5 values for discrete curvature as $\left\{-1 / \rho_{i},-1 /\left(2 \rho_{i}\right), 0,1 /\left(2 \rho_{i}\right), 1 / \rho_{i}\right\}$ also, $\delta_{1}=4 \mathrm{sec}$ and $\delta_{2}=1.5 \mathrm{sec}$. The tessellation of the field is made by 127 circles with radius $r=20 \mathrm{~m}$.

Figure 5 shows the generated trajectories for the vehicles covering a civilian neighborhood area bounded by a rectangular region. We also used hardware in the loop simulations to verify the generated trajectories Fig. 6. Finally we implemented the coverage algorithm using the GRASP lab's UAVs. The picture were taken with the frequency of $1 \mathrm{sec}$. Figure 7 shows the coverage of the area using the taken pictures in two different $15 \mathrm{sec}$. time periods. The pictures were stitched using Autostitch tools [23].

For solving the IP we used mixed randomized and heuristic search based techniques. The execution time for generating $300 \mathrm{sec}$. (solving IP 20 times) trajectories for the UAVs is 7 sec. on a $2 \mathrm{GHz}, 768 \mathrm{MB}$ Pentium 4 laptop.

\section{CONCLUSION}

In this paper we presented a path planning algorithm for time sensitive cooperative surveillance using UAVs equipped with fixed cameras. The main challenges were to generate feasible trajectories while incorporating the complexity and coupling of the camera fields of view and flight paths. Future research directions will include (3-d) path planning using Serret-Frenet equations and planning using both the curvature and torsion functions, also adding collision and obstacle avoidance to our current method. 


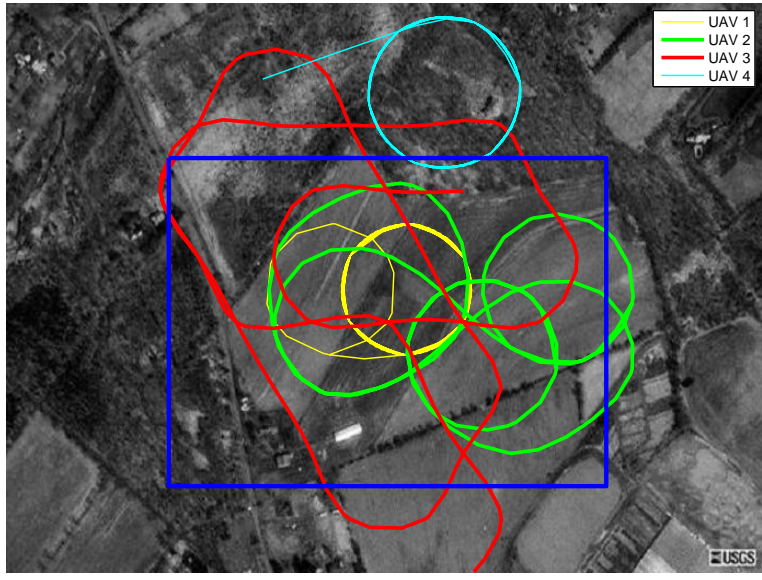

Fig. 5. $300 \mathrm{sec}$ generated trajectories for all UAVs

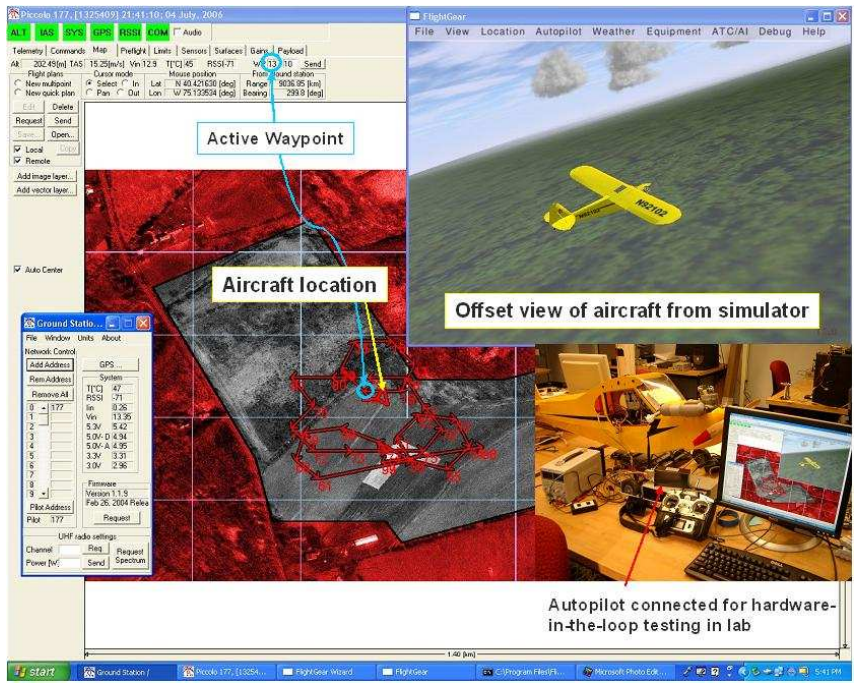

Fig. 6. Hardware in the loop simulations

\section{ACKNOWLEDGMENTS}

The first author would like to thank Michael Zavlanos for insightful discussions on the subject of this paper.

\section{REFERENCES}

[1] S. Bayraktar, G. Fainekos and G. Pappas, Experimental Cooperative Control of Fixed-Wing Unmanned Aerial Vehicles, In the Proceedings of the 43rd IEEE Conference on Decision and Control, The Bahamas, December 2004

[2] H.J. Kim, D.H. Shim, S. Sastry, Flying robots: modeling, control and decision making, in Proceedings of International Conference on Robotics and Automation, ICRA 02, 11-15 May 2002

[3] E. Olsen, C.W. Park, and J. How, 3D Formation Flight using Differential Carrier-phase GPS Sensors, The J. of The Institute Of Navigation, Spring, 1999, Vol. 146, No. 1, pp. 3548.

[4] J. Evans, G. Inalhan, J.S. Jang, R. Teo, C.J. Tomlin, DragonFly: a versatile UAV platform for the advancement of aircraft navigation and control, in 20th Conference Digital Avionics Systems, 14-18 Oct. 2001

[5] Fierro, R., Belta, C., Desai, J.P. and Kumar, V., On Controlling Aircraft Formations, Proceedings of the 40th IEEE Conference on Decision and Control, Orlando, FL, Dec. 2001, vol. 2, pp. 1065-70.

[6] L. E. Dubins, On curves of minimal length with a constraint on average curvature, and with prescribed initial and terminal positions and tangents, Amer. J. Math., vol. 79, no. 3, pp. 497-516, 1957

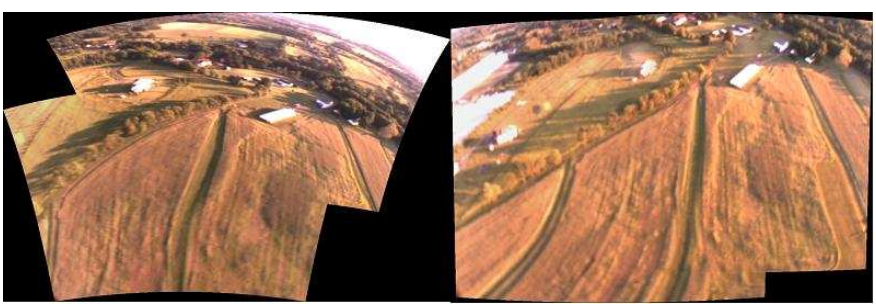

Fig. 7. Two samples of tesselated coverage area

[7] K. Savla, E. Frazzoli, and F. Bullo. On the point-to-point and traveling salesperson problems for Dubins' vehicles. In proceedings of the American Control Conference, 2005.

[8] S. Rathinam, R. Sengupta, A Safe Flight Algorithm for Unmanned Aerial Vehicles, in IEEE Aerospace Conference, Montana, 2004

[9] S. Rathinam, M. Zennaro, T. Mak and R. Sengupta, An architecture for UAV team control, Proc. IFAC Conference on Intelligent Autonomous Vehicles in Portugal, July 2004.

[10] A. Ahmadzadeh, B. Sayyar-Roudsari and A. Homaifar, A Hybrid Evolutionary-Gradient Search Algorithm for Capacitated Multi-Source Multi-UAVs Scheduling with Time Windows, Recent Developments in Cooperative Control and Optimization Edt. S. Butenko, R. Murphey, and M. Pardalos, Kluwer Academic Publishers, December 2003

[11] A. Richards, J. How, Mixed-integer Programming for Control, In proceedings of the American Control Conference,June 2005.

[12] H. Choset, Coverage for robotics-a survey of recent results, Annals of Mathematics and Artificial Intelligence Vol. 31 , pp. 113-126, 2001

[13] D. A. Castanon, Approximated dynamic programming for sensor management, in Proc. IEEE Conf. Decision Control, San Diego, CA, pp. 1202-1207. Dec. 1997

[14] D. J. Cool, P. Gmytrasiewicz, and L. B. Holder, Decision-theoretic cooperative sensor planning, IEEE Trans. Pattern Anal. Machine Intell., vol. 18 , no. 10 , pp. 892-902, Oct. 1996.

[15] M. Kalandros, L. Y. Pao, and Y. Ho, "Randomization and superheuristics in choosing sensor sets for target tracking applications, in Proc. IEEE Conf. Decision Control, vol. 2, Phoenix, AZ, pp. 1803-1808. Dec. 1999

[16] P. Vanheeghe, E. Duflos, P. E. Dumont, and V. Nimier, Sensor management with respect to danger level of targets, in Proc. IEEE Conf. Decision Control, vol. 5, Orlando, FL, pp. 4439-4444, 2001

[17] J. Cortes, S. Martinez, T. Karatas, and F. Bullo, Coverage control for mobile sensing networks. In Proceedings of IEEE International Conference on Robotics and Automation, 2002, pp. 1327-1332.

[18] J.J. Enright and E. Frazzoli and K. Savla and F. Bullo. On Multiple UAV Routing with Stochastic Targets: Performance Bounds and Algorithms. Proc. of the AIAA Conf. on Guidance, Navigation, and Control, August 2005.

[19] Do Carmo M.P., Differential geometry of curvs and surfaces, Prentice Hall, 1976.

[20] R. Kershner, The number of circles covering a set. Amer. J. Math., vol. 61, pp. 665-671, 1939.

[21] Jadbabaie, A., Yu, J., and Hauser, J., Unconstrained receding-horizon control of nonlinear systems,IEEE Transactions on Automatic Control, Vol. 46, No. 5, May 2001, pp. 776-783.

[22] S. M. LaValle. Planning Algorithms. [Online], 2004. Available at http://msl.cs.uiuc.edu/planning/.

[23] Autostitch tools.

Available online at www.cs.ubc.ca/ mbrown/autostitch/autostitch.htm 